\title{
KERNEL REGRESSION, MINIMAX RATES AND EFFECTIVE DIMENSIONALITY: BEYOND THE REGULAR CASE
}

\author{
GILLES BLANCHARD AND NICOLE MÜCKE
}

\begin{abstract}
We investigate if kernel regularization methods can achieve minimax convergence rates over a source condition regularity assumption for the target function. These questions have been considered in past literature, but only under specific assumptions about the decay, typically polynomial, of the spectrum of the the kernel mapping covariance operator. In the perspective of distribution-free results, we investigate this issue under much weaker assumption on the eigenvalue decay, allowing for more complex behavior that can reflect different structure of the data at different scales.
\end{abstract}

\section{INTRODUCTION AND MOTIVATION}

1.1. Kernel regression. We are concerned with the classical regression learning problem, where we observe training data $\mathcal{D}:=\left(X_{i}, Y_{i}\right)_{i=1, \ldots, n}$, assumed to be an i.i.d. sample from a distribution $\mathbb{P}$ over $X \times \mathbb{R}$ ( $\nu$ will denote the marginal distribution of $X$ ), and the goal is to estimate the regression function $f^{*}(x):=\mathbb{E}[Y \mid X=x]$. We consider the well-established setting of (reproducing) kernel learning: we assume a positive semi-definite kernel $k(.,$. has been defined on $\mathcal{X}$, with associated canonical feature mapping $\Phi: \mathcal{X} \rightarrow \mathcal{H}$ into a corresponding reproducing kernel Hilbert space $\mathcal{H}$. A classical methodology to handle this problem is kernel ridge regression, depending on a regularization parameter $\lambda>0$, giving rise to the estimate $\widehat{f}_{\lambda}$; we will consider a much larger class of kernel methods below, but restrict ourselves to that particular example for the sake of the present introduction.

A common goal of learning theory is to give upper bounds for the convergence of $\widehat{f}_{\lambda}$ to $f^{*}$, and derive rates of convergence (as $n \rightarrow \infty$ ) under appropriate assumptions on the "regularity" of $f^{*}$. In this paper, te notion of convergence we will consider is the usual squared $L^{2}(\nu)$ distance with respect to the sampling distribution, $\left\|\widehat{f}_{\lambda}-f^{*}\right\|_{2, \nu}^{2}=$ $\mathbb{E}\left[\left(\widehat{f}_{\lambda}(X)-f^{*}(X)\right)^{2}\right]$; it is well-known that this is equal to the excess risk with respect to Bayes when using the squared loss, i.e.

$$
\left\|\widehat{f}_{\lambda}-f^{*}\right\|_{2, \nu}^{2}=\mathbb{E}\left[\left(Y-\widehat{f}_{\lambda}(X)\right)^{2}\right]-\min _{f: X \rightarrow \mathbb{R}} \mathbb{E}\left[(Y-f(X))^{2}\right] .
$$

More precisely, we are interested in bounding the averaged above error over the draw of the training data (this is also called Mean Integrated Squared Error or MISE in the statistics literature). We will also consider convergence estimates in the (stronger) averaged $\mathcal{H}$-norm.

Date: November 15, 2016. 
1.2. Minimax error in classical nonparametrics. When upper bounds or convergence rates for a specific method are obtained, it is natural to ask to what extent they can be considered optimal. The classical yardstick is the notion of minimax error over a set of candidate $\mathcal{P}$ (hypotheses) for the data generating distribution:

$$
\mathcal{R}(\mathcal{P}, n):=\inf _{\widehat{f}} \sup _{\mathbb{P} \in \mathcal{P}} \mathbb{E}_{\mathcal{D} \sim \mathbb{P}^{\otimes}}\left[\left\|\widehat{f}-f_{\mathbb{P}}^{*}\right\|_{2, \nu}^{2}\right],
$$

where the inf operation is over all estimators, and we added an index $\mathbb{P}$ to $f^{*}$ to emphasize its dependence on the data generating distribution.

In the nonparametric statistics literature, it is commonly assumed that $X$ is some compact set of $\mathbb{R}^{d}$, the sampling distribution $\nu$ has an upper bounded density with respect to the Lebesgue measure, and the type of regularity considered for the target function is a Sobolev-type regularity, i.e., that the target function $f^{*}$ has squared-integrable $r$-th derivative. This is equivalent to say that $f^{*}$ belongs to a Sobolev ellipsoid of radius $R$,

$$
f^{*} \in\left\{f: \sum_{i \geq 1} i^{-\frac{2 r}{d}} f_{i}^{2} \leq R^{2}\right\}
$$

where $f_{i}$ denotes the coefficients of $f$ in a (multidimensional) trigonometric function basis. Minimax rates in such context are known to be of the order $\mathcal{O}\left(n^{-\frac{2 r}{2 r+d}}\right)$, and can be attained for a variety of classical procedures [11, 12].

1.3. Minimax error in a distribution-free context. In the statistical learning context, the above assumptions are unsatisfying. The first reason is that learning using kernels is often applied to non-standard spaces, for instance graphs, text strings, or even probability distributions (see, e.g., [4]). There is often no "canonical" notion of regularity of a function on such spaces, nor a canonical reference measure which would take the role of the Lebesgue measure in $\mathbb{R}^{d}$. The second reason is that learning theory focuses on a distribution-free approach, i.e., avoiding specific assumptions on the generating distribution. By contrast, it is a very strong assumption to posit that the sampling distribution $\nu$ is dominated by some reference measure (be it Lebesgue or otherwise), especially for non-standard spaces, or in $\mathbb{R}^{d}$ if the dimension $d$ is large. In the latter case, the convergence rate $\mathcal{O}\left(n^{-\frac{2 r}{2 r+d}}\right)$ becomes very slow (curse of dimensionality), yet it is often noticed in practice that many kernel-based methods perform well. The reason is that for high-dimensional data, more often than not the sampling distribution $\nu$ is actually concentrated on some lower-dimensional structure, so that the assumption of $\nu$ having bounded density in $\mathbb{R}^{d}$ is violated: convergence rates could then be much faster. For these reasons, it has been proposed to consider regularity classes for the target function having a form similar to (1.2), but reflecting implicitly the geometry corresponding to the choice of the kernel and to the sampling distribution. More precisely, denote $B_{\nu}$ (uncentered) covariance operator of the kernel feature mapping $\Phi(X)$ and $\left(\mu_{\nu, i}, \psi_{\nu, i}\right)_{i \geq 1}$ an eigendecomposition of $B_{\nu}$. For $r, R>0$, introduce the class

$$
\Omega(\nu, r, R):=\left\{f \in \mathcal{H}: \sum_{i \geq 1} \mu_{\nu, i}^{r} f_{i}^{2} \leq R^{2}\right\}=B_{\nu}^{r} B(\mathcal{H}, R),
$$


where $B(\mathcal{H}, R)$ is the ball of radius $R$ in $\mathcal{H}$, and $f_{i}:=\left\langle f, \psi_{\nu, i}\right\rangle$ are the coefficients of $f$ in the eigenbasis. Clearly, (1.3) has a form similar to (1.2), but in a basis and scaling that reflects the properties of the distribution of $\Phi(X)$. If the target function $f^{*}$ is well approximated in this basis in the sense that its coefficients decay fast enough in comparison to the eigenvalues, it is considered as regular in this geometry (higher regularity corresponds to higher values of $r$ and/or lower values of $R$ ). This type of regularity class, also called source condition, have been considered in a statistical learning context by [5], and [6] have established upper bounds for the performance of kernel ridge regression $\widehat{f}_{\lambda}$ over such classes; this has been extended to other types of kernel regularization methods by [2, 8, 1]. These bounds rely on tools introduced in the seminal work of [13], and depend in particular on the notion of effective dimension of the data with respect to the regularization parameter $\lambda$, defined as

$$
\mathcal{N}(\lambda):=\operatorname{tr}\left(\left(B_{\nu}+\lambda\right)^{-1} B_{\nu}\right)=\sum_{i \geq 1} \frac{\mu_{\nu, i}}{\mu_{\nu, i}+\lambda} .
$$

As before, the next question of importance is whether such upper bounds can be proved to be minimax optimal over the classs $\Omega(\nu, r, R)$, assuming the regularization parameter $\lambda$ is tuned appropriately. This question has been answered positively when a polynomial decay of the eigenvalues, $\mu_{v, i} \asymp i^{-b}$, is assumed ( $\asymp$ stands for upper and lower bounded up to a constant). In this case $\mathcal{N}(\lambda)$ can be evaluated, and for an appropriate choice of $\lambda$, the upper bound can be matched by a corresponding lower bound. The first comprehensive result in this direction was established by [6] ; 1] give a sharp estimate of the convergence rate in this case including the dependence on the parameters $R$ and noise variance $\sigma$, namely $\mathcal{O}\left(R^{2}\left(\frac{\sigma^{2}}{R^{2} n}\right)^{\frac{2 r+1}{2 r+1+1 / b}}\right)$.

1.4. Contributions: beyond regular decay of eigenvalues. The assumption of polynomially decaying eigenvalues yields explicit minimax convergence rates, relates closely (1.3) to (1.2), and ensures that kernel methods can achieve those optimal rates. Yet, once again it is unsatisfying from a distribution-free point of view. Remember the stucture of the eigenvalues reflects the covariance of the feature mapping $\Phi(X)$; for complex data, there is no strong reason to expect that their decay should be strictly polynomial, and we would like to cover behavior as general as possible for the eigenvalue decay, for instance:

- decay rates including other slow varying functions, such as $\mu_{v, i} \asymp i^{-b}(\log i)^{c}(\log \log i)^{d}$;

- eigenvalue sequence featuring plateaus separated by relative gaps;

- shifting of switching along the sequence between different polynomial-type regimes,

which all might correspond to diffent type of structure of the data at different scales. With the distribution-free point of view in mind, we therefore try to characterize minimax rates for target function classes of the form (1.3), striving for assumptions as weak as possible on the eigenvalue sequence. More importantly, we investigate if kernel methods also achieve minimax rates in this general case. 


\section{SetTing}

We let $\mathcal{Z}=\mathcal{X} \times \mathcal{R}$ denote the sample space, where the input space $\mathcal{X}$ is a standard Borel space endowed with a fixed unknown probability measure $\nu$. The kernel space $\mathcal{H}$ is assumed to be separable, equipped with a measurable positive semi-definite kernel $k$, bounded by $\kappa$, implying continuity of the inclusion map $I_{\nu}: \mathcal{H} \longrightarrow L^{2}(\nu)$. Moreover, we consider the covariance operator $B_{\nu}=I_{\nu}^{*} I_{\nu}=\mathbb{E}[\Phi(X) \otimes \Phi(X)]$, which can be shown to be positive self-adjoint trace class (and hence is compact). Given a sample $\mathbf{x}=\left(x_{1}, \ldots, x_{n}\right) \in X^{n}$, we define the sampling operator $S_{\mathbf{x}}: \mathcal{H} \longrightarrow \mathcal{R}^{n}$ by $\left(S_{\mathbf{x}} f\right)_{i}=\left\langle f, \Phi\left(x_{i}\right)\right\rangle_{\mathcal{H}}$. The empirical covariance operator is given by $B_{\mathbf{x}}=S_{\mathbf{x}}^{*} S_{\mathbf{x}}$. Throughout we denote by $\mu_{j}$ the positive eigenvalues of $B_{\nu}$ in decreasing order, satisfying $0<\mu_{j+1} \leq \mu_{j}$ for all $j>0$ and $\mu_{j} \searrow 0$. For any $t>0$ we let

$$
\mathcal{F}(t):=\#\left\{j \in \mathbb{N}: \mu_{j} \geq t\right\} .
$$

Note that $\mathcal{F}$ is left-continuous and decreasing as $t$ grows with $\mathcal{F}(t) \equiv 0$ for any $t>\kappa^{2}$, and $\mathcal{F}$ has limit $+\infty$ in $0^{+}$. Given $r>0$, we set $\mathcal{G}(t):=\frac{t^{2 r+1}}{\mathcal{F}(t)}$ (possibly taking the value $\infty$ if $\mathcal{F}(t)=0)$, which is left-continous and increasing on $\left(0, \kappa^{2}\right]$ with $\mathcal{G}\left(0^{+}\right)=0$. Define the generalized inverse for any $u>0$ by

$$
\mathcal{G}^{-1}(u):=\max \{t: \mathcal{G}(t) \leq u\} .
$$

Some properties of $\mathcal{F}, \mathcal{G}$ and $\mathcal{G}^{-1}$ are collected in Lemma 5.1.

In order to present our main results, we require some assumptions on the learning problem:

(1) Noise Assumption: The sampling is assumed to be random i.i.d., where each observation point $\left(X_{i}, Y_{i}\right)$ follows the model $Y=f(X)+\varepsilon$. We will make the following Bernstein-type assumption on the observation noise distribution: For any integer $m \geq 2$ and some $\sigma>0$ and $M>0$ :

$\operatorname{Bernstein}(M, \sigma): \mathbb{E}\left[\varepsilon^{m} \mid X\right] \leq \frac{1}{2} m ! \sigma^{2} M^{m-2} \quad \nu-$ a.s. .

(2) Assumption on eigenvalues: For any $j$ sufficiently large and some $\nu_{*} \geq \nu^{*} \geq 1$

$\left(\operatorname{Eigen}^{<}\left(\nu^{*}\right)\right): \mu_{2 j} / \mu_{j} \leq 2^{-\nu^{*}}, \quad\left(\operatorname{Eigen}^{>}\left(\nu_{*}\right)\right): \mu_{2 j} / \mu_{j} \geq 2^{-\nu_{*}}$.

(3) The regularity of the target function $f_{\mathbb{P}}^{*}$ is measured in terms of a source condition: $\mathbf{S C}(r, R): f_{\mathbb{P}}^{*} \in B_{\nu}^{r} B(\mathcal{H}, R)$, where $r>0, R>0$.

(4) Model: Let $\theta=(M, \sigma, R) \in \mathcal{R}_{+}^{3}$. The class $\mathcal{M}_{\theta}:=\mathcal{M}(\theta, r, \nu)$ consists of all distributions $\mathbb{P}$ with fixed $X$-marginal $\nu$ and conditional distribution of $Y$ given $X$ satisfying $\operatorname{Bernstein}(M, \sigma)$ for the deviations and $\mathbf{S C}(r, R)$ for the mean.

(5) Regularization: We find our estimator for $f_{\mathbb{P}}^{*}$ via some linear spectral regularization function $g_{\lambda}:[0,1] \longrightarrow \mathcal{R}$, satisfying the following conditions for any $0<\lambda \leq 1$ :

$$
\sup _{0<t \leq 1}\left|t g_{\lambda}(t)\right| \leq D, \quad \sup _{0<t \leq 1}\left|g_{\lambda}(t)\right| \leq \frac{E}{\lambda}, \quad \sup _{0<t \leq 1}\left|r_{\lambda}(t)\right| \leq \gamma_{0},
$$


where $r_{\lambda}(t)=1-g_{\lambda}(t) t$, for some $D<\infty, E<\infty$ and $\gamma_{0}<\infty$. The qualification of the regularization $\left\{g_{\lambda}\right\}_{\lambda}$ is the maximal $q$ such that for any $0<\lambda \leq 1$

$$
\sup _{0<t \leq 1}\left|r_{\lambda}(t)\right| t^{q} \leq \gamma_{q} \lambda^{q}
$$

for some constant $\gamma_{q}>0$.

These conditions originally characterize regularization methods for finding stable solutions for ill-posed inverse problems ([9]). It has been shown in e.g. [10] and [7] that precisely these methods are very well applicable in the context of learning theory.

Given a sample $\mathbf{z}=(\mathbf{x}, \mathbf{y}) \in(X \times \mathcal{R})^{n}$, we define the estimator $f_{\mathbf{z}}^{\lambda}$ for a suitable a-priori parameter choice $\lambda=\lambda_{n}$ by

$$
f_{\mathbf{z}}^{\lambda_{n}}:=g_{\lambda_{n}}\left(\kappa^{-2} B_{\mathbf{x}}\right) \kappa^{-2} S_{\mathbf{x}}^{\star} \mathbf{y}=g_{\lambda_{n}}\left(\bar{B}_{\mathbf{x}}\right) \bar{S}_{\mathbf{x}}^{\star} \mathbf{y}
$$

where we have introduced the shortcut notation $\bar{B}_{x}:=\kappa^{-2} B_{\mathbf{x}}$ and $\bar{S}_{\mathbf{x}}:=\kappa^{-2} S_{\mathbf{x}}$.

\section{MAIN RESULTS}

Under the assumptions of the previous section, we establish that the order of the minimax rates is given by the following quantities:

$$
a_{n, \theta}^{\mathcal{H}}:=R \mathcal{G}^{-1}\left(\frac{\sigma^{2}}{R^{2} n}\right)^{r}, \quad a_{n, \theta}^{L^{2}(\nu)}:=R \mathcal{G}^{-1}\left(\frac{\sigma^{2}}{R^{2} n}\right)^{r+\frac{1}{2}}
$$

Theorem 3.1 (Minimax lower rate). Let $r>0$ be fixed and assume $\nu$ satisfies (Eigen $\left.{ }^{>}\left(\nu_{*}\right)\right)$. Then the sequence $\left(a_{n, \theta}^{\mathcal{H}}\right)_{n}$ defined in (3.1) is a minimax lower rate of convergence for the RKHS-norm $\mathcal{H}$, for the model family $\mathcal{M}_{\theta}:=\mathcal{M}(\theta, r), \theta:=(M, \sigma, R) \in \mathcal{R}_{+}^{3}$, i.e. there exists $\tau>0$ (possibly depending on $r$ and $\nu_{*}$, but not on $\theta$ ) such that

$$
\liminf _{n \rightarrow \infty} \inf _{f_{\bullet}} \sup _{\mathbb{P} \in \mathcal{M}_{\theta}} \mathbb{P}^{\otimes n}\left(\left\|f_{\mathbb{P}}^{*}-f_{\mathbf{z}}\right\|_{\mathcal{H}}>\tau a_{n, \theta}^{\mathcal{H}}\right)>0,
$$

where the infimum is taken over all estimators. Similarly, (3.2) also holds w.r.t. to $L^{2}(\nu)$ norm with minimax lower rate $a_{n, \theta}^{L^{2}(\nu)}$ over the same model family.

Theorem 3.2 (Upper rate). Assume $\mathbf{S C}(r, R)$, $\operatorname{Bernstein}(M, \sigma)$ and that $\nu$ satisfies (Eigen $\left.{ }^{<}\left(\nu^{*}\right)\right)$. Consider the model $\mathcal{M}_{\theta}$, where $\theta:=(M, \sigma, R) \in \mathcal{R}_{+}^{3}$. Given a sample $\mathbf{z} \in Z^{n}$, define $f_{\mathbf{z}}^{\lambda}$ as in (2.3), using a regularization function of qualification $q \geq r$, with the parameter sequence

$$
\lambda_{n, \theta}=\min \left(\mathcal{G}^{-1}\left(\frac{\sigma^{2}}{R^{2} n}\right), 1\right) .
$$

Then there exists $n_{0}>0$ (depending on the above parameters) such that for any $n \geq n_{0}$ with probability at least $1-\eta$ :

$$
\left\|f_{\mathbb{P}}^{*}-f_{\mathbf{z}}^{\lambda_{n}}\right\|_{\mathcal{H}} \leq C_{r, \nu^{*}} \log \left(8 \eta^{-1}\right) a_{n, \theta}^{\mathcal{H}},
$$


provided $\log \left(8 \eta^{-1}\right) \leq C_{r, \nu^{*}} \lambda_{n}^{-r}$. Similarly, the sequence $a_{n, \theta}^{L^{2}(\nu)}$ also fulfills (3.4) in $L^{2}(\nu)$ norm with identical parameter sequence (3.3), using a regularization function with qualification $q \geq r+\frac{1}{2}$.

Remark: In the above Theorem, $n_{0}$ can possibly depend on all parameters, but the constant $C_{r, \nu^{*}}$ in front of the upper bound does not depend on $R, \sigma$, nor $M$. In this sense, this result tracks precisely the effect of these important parameters on the scaling of the rate, but remains asymptotic in nature: it cannot be applied if, say, $R, \sigma$ of $M$ also depend on $n$ (because the requirement $n \geq n_{0}$ might then lead to an impossiblity.)

\section{Discussion}

Range of applications. The assumptions we made on the spectrum decay, namely (Eigen $^{<}\left(\nu^{*}\right)$ ) and (Eigen $^{>}\left(\nu_{*}\right)$ ), are much weaker than the usual assumptions of polynomial decay on the eigenvalues. Therefore, our results establish that in this much broader situation, usual kernel regularization methods can achieve minimax rates over the regularity classes $\mathbf{S C}(r, R)$. In particular, these conditions accomodate for changing behavior of the spectrum at different scales, as well as other situations delineated in the introduction. Still, our conditions do not encompass totally arbitary sequences: (Eigen ${ }^{>}\left(\nu_{*}\right)$ ) in particular implies that the eigenvalues cannot decrease with a polynomial rate with exponent larger than $\nu_{*}$. While the latter constant can be chosen arbitrary large and only results in a change of constant factor in the rates, it excludes for example exponentially decreasing eigenvalues. It is not clear whether such sharp results about classes $\mathbf{S C}(r, R)$ can be established in this case. However, we observe that in such cases (typically observed when using the Gaussian kernel), in practice the kernel parameters (e.g. the bandwith) are also tuned in addition to $\lambda$, which might reflect the fact that for badly tuned bandwidth of the kernel, tuning of the regularization parameter $\lambda$ alone might not give satisfactory (minimax) results. On the other hand, the results we obtained might provide an additional motivation for using "rougher" kernels than Gaussian, leading to a softer decay of eigenvalues, in which case minimax adaptivity is at hand over a large regularity class. The latter is true however only if the qualification of the method is large, which is not the case for the usual kernel ridge regression: hence, rougher kernels should be used with methods having a large qualification (for instance $L^{2}$ boosting).

Adaptivity. Our results establish the existence of a suitable regularization parameter $\lambda$ such that the associated estimator attains the minimax rate if the regularity class parameter are known in advance. The latter is of course not realistic, but in the case of $L^{2}$ (prediction) error, the principle of using a grid for $\lambda$ and then using a hold-out sample to select a value of $\lambda$ from the data is known to be able to select a value close to the optimal choice in a broad domain of situations (see, for instance, [3]), so that we can turn our results to datadependent minimax adaptivity even in the absence of a priori knowledge of the regularity parameters. 


\section{ProOFS}

The proof of Theorem 3.2 and Theorem 3.1 will be given not only in both $\mathcal{H}$ - norm and $L^{2}(\nu)$-norm, but also for all intermediate norms, namely for $\|f\|_{s}:=\left\|B^{s} f\right\|_{\mathcal{H}}$, where $s \in[0,1 / 2]$. Note that $s=0$ corresponds to $\mathcal{H}$ - norm, while $s=1 / 2$ corresponds to $L^{2}(\nu)$-norm.

To simplify notation we will adopt the following conventions: The dependence of multiplicative constants $C$ on various parameters will (generally) be omitted, except for $\sigma, M, R, \eta$ and $n$ which we want to track precisely. The expression "for $n$ big enough" means that the statement holds for $n \geq n_{0}$, with $n_{0}$ potentially depending on all model parameters (including $\sigma, M$ and $R$ ), but not on $\eta$.

5.1. Preliminaries. We start by collecting some useful properties for the functions $\mathcal{F}$ and $\mathcal{G}$ in the following lemma.

Lemma 5.1. (1) Let $c \leq 1$ be fixed. Then for any $t$,

$$
\mathcal{G}(c t) \leq c \mathcal{G}(t)
$$

(2) Assume (Eigen $\left.{ }^{<}\left(\nu^{*}\right)\right)$ holds. Let $C \geq 1$ be fixed. Then for any $t$ small enough,

$$
\mathcal{F}(t) \leq 4 C^{\frac{1}{\nu^{*}}} \mathcal{F}(C t) \quad \text { and } \quad \mathcal{G}(C t) \leq 4 C^{2 r+1+\frac{1}{\nu^{*}}} \mathcal{G}(t) .
$$

(3) Assume $\left(\right.$ Eigen $\left.^{<}\left(\nu^{*}\right)\right)$ holds. For any $u>0$ it holds $\mathcal{G}\left(\mathcal{G}^{-1}(u)\right) \leq u$ and for $u$ small enough,

$$
\mathcal{G}\left(\mathcal{G}^{-1}(u)\right) \geq \frac{u}{4}
$$

Proof of Lemma 5.1. For point 1 of the Lemma, let $c \leq 1$ be fixed; just write by definition of $\mathcal{G}$ and the fact that $\mathcal{F}$ is nonincreasing:

$$
\mathcal{G}(c t)=\frac{c^{2 r+1} t^{2 r+1}}{\mathcal{F}(c t)} \leq c^{2 r+1} \mathcal{G}(t) \leq c \mathcal{G}(t) .
$$

For point 2 , let $j_{0} \geq 1$ such that $\left(\operatorname{Eigen}^{<}\left(\nu^{*}\right)\right)$ holds for any $j \geq j_{0}$ and let $t_{0}$ be small enough such that $\mathcal{F}\left(t_{0}\right) \geq j_{0}$.

Let $C \geq 1$ be fixed and $t \leq C^{-1} t_{0}$, so that $k_{t}:=\mathscr{F}(C t) \geq j_{0}$. By definition $\mu_{k_{t}+1}<C t$. Furthermore for any $i \geq 1$ we have $\mu_{2^{i}\left(k_{t}+1\right)} \leq 2^{-i \nu^{*}} \mu_{\left(k_{t}+1\right)}$ by repeated application of (Eigen $^{<}\left(\nu^{*}\right)$ ). Choosing $i:=1+\left\lfloor\frac{\log _{2} C}{\nu^{*}}\right\rfloor$, we have $2^{-i \nu^{*}} C \leq 1$ and $2^{i} \leq 2 C \frac{1}{\nu^{*}}$. Combining the first inequality with what precedes we deduce $\mu_{2^{i}\left(k_{t}+1\right)}<t$ and thus $\mathcal{F}(t) \leq 2^{i}\left(k_{t}+1\right)-$ $1 \leq 4 C^{\frac{1}{\nu^{*}}} \mathcal{F}(C t)$. We deduce

$$
\mathcal{G}(C t)=\frac{C^{2 r+1} t^{2 r+1}}{\mathcal{F}(C t)} \leq \frac{4 C^{2 r+1+\frac{1}{\nu^{*}}} t^{2 r+1}}{\mathcal{F}(t)}=4 C^{2 r+1+\frac{1}{\nu^{*}}} \mathcal{G}(t) .
$$

We turn to point 3. Since $\mathcal{G}$ is left-continuous, the supremum in the definition (2.2) of its inverse $\mathcal{G}^{-1}$ is indeed a maximum (also the set over which the max is taken is nonempty since $\mathcal{G}\left(0^{+}\right)=0$ and $\left.u>0\right)$, and therefore must satisfy $\mathcal{G}\left(\mathcal{G}^{-1}(u)\right) \leq u$.

Consider now $\mathcal{F}\left(t^{+}\right):=\#\left\{j \in \mathbb{N}: \mu_{i}>t\right\}$, let $t_{0}^{\prime}$ be small enough such that $\mathcal{F}\left(t_{0}^{\prime}\right) \geq$ $2 j_{0}$, and assume $t<\min \left(t_{0}^{\prime}, \mu_{1}\right)$. The second component of the latter minimum ensures 
$\mathcal{F}\left(t^{+}\right) \geq 1$. If $t \notin\left\{\mu_{i}, i \geq 1\right\}$, then $\mathcal{F}$ is continous in $t$ and $\mathcal{F}(t)=\mathscr{F}\left(t^{+}\right)$. Otherwise, $t=\mu_{k}$ with $k=\mathcal{F}(t) \geq 2 j_{0}$, so that $\frac{\mu_{k}}{\mu_{\left\lfloor\frac{k}{2}\right\rfloor}} \leq 2^{-\nu^{*}}<1$, that is to say $t<\mu_{\left\lfloor\frac{k}{2}\right\rfloor}$, implying $F\left(t^{+}\right) \geq\left\lfloor\frac{k}{2}\right\rfloor \geq \frac{1}{2} \mathcal{F}(t)-1$ and finally $\mathcal{F}(t) \leq 4 \mathcal{F}\left(t^{+}\right)$.

Consider now $u$ small enough such that $t=\mathcal{G}^{-1}(u)<\min \left(t_{0}^{\prime}, \mu_{1}\right)$ as above. Then $\mathcal{G}\left(t^{+}\right) \geq u$ and

$$
\mathcal{G}\left(\mathcal{G}^{-1}(u)\right)=\mathcal{G}(t)=\frac{t^{2 r+1}}{\mathcal{F}(t)} \geq \frac{1}{4} \frac{t^{2 r+1}}{\mathcal{F}\left(t^{+}\right)}=\frac{1}{4} \mathcal{G}\left(t^{+}\right) \geq \frac{u}{4}
$$

Lemma 5.2 (Effective dimensionality). Assume (Eigen $\left.{ }^{<}\left(\nu^{*}\right)\right)$ holds. The effective dimension (defined in (1.4)) satisfies for any $\lambda$ sufficiently small and some $C_{\nu^{*}}<\infty$

$$
\mathcal{N}(\lambda) \leq C_{\nu^{*}} \mathcal{F}(\lambda) \text {. }
$$

Proof of Lemma 5.2. Let $j_{0} \geq 1$ such that $\left(\right.$ Eigen $\left.^{<}\left(\nu^{*}\right)\right)$ holds for any $j \geq j_{0}$ and let $\lambda_{0}$ be small enough such that $\mathcal{F}\left(\lambda_{0}\right) \geq j_{0}$. For $\lambda \leq \lambda_{0}$, denote $j_{\lambda}:=\mathscr{F}(\lambda)$. Then, using $\mu_{j}<\mu_{j}+\lambda$ and $\lambda<\mu_{j}+\lambda$, we obtain

$$
\mathcal{N}(\lambda)=\sum_{j=1}^{j_{\lambda}} \frac{\mu_{j}}{\mu_{j}+\lambda}+\sum_{j>j_{\lambda}} \frac{\mu_{j}}{\mu_{j}+\lambda} \leq j_{\lambda}+\frac{1}{\lambda} \sum_{j>j_{\lambda}} \mu_{j} .
$$

Focussing on the tail sum we see that

$$
\begin{aligned}
\sum_{j \geq j_{\lambda}} \mu_{j}=\sum_{l=0}^{\infty} \sum_{j=\left(j_{\lambda}+1\right) 2^{l}}^{\left(j_{\lambda}+1\right) 2^{l+1}-1} \mu_{j} \leq\left(j_{\lambda}+1\right) \mu_{j_{\lambda}+1} \sum_{l=0}^{\infty} 2^{l} 2^{-l \nu *} & =\left(j_{\lambda}+1\right) \mu_{j_{\lambda}+1}\left(1-2^{1-\nu *}\right)^{-1} \\
& \leq \lambda(\mathcal{F}(\lambda)+1)\left(1-2^{1-\nu *}\right)^{-1}
\end{aligned}
$$

where the first inequality comes from the fact that the sequence $\left(\mu_{j}\right)_{j}$ is decreasing and by repeated application of $\left(\right.$ Eigen $\left.^{<}\left(\nu^{*}\right)\right)$; and the second inequality from the definition of $j_{\lambda}$. Collecting all ingredients we obtain for any $\lambda \leq \lambda_{0}$ :

$$
\mathcal{N}(\lambda) \leq \mathcal{F}(\lambda)\left(1+2\left(1-2^{1-\nu *}\right)^{-1}\right) .
$$

5.2. Proof of upper rate. The proof of Theorem 3.2 relies on the following non-asymptotic result, given in [1]:

Proposition 5.3. Let $s \in\left[0, \frac{1}{2}\right]$, assume $\mathbf{S C}(r, R)$ and $\operatorname{Bernstein}(M, \sigma)$. Let $f_{\mathbf{z}}^{\lambda}$ be defined as in (2.3) using a regularization function of qualification $q \geq r+s$. Then, for any $\eta \in(0,1), \lambda \in(0,1]$ and $n \in \mathbb{N}$ satisfying

$$
n \geq 64 \lambda^{-1} \max (\mathcal{N}(\lambda), 1) \log ^{2}(8 / \eta),
$$

we have with probability at least $1-\eta$ :

$$
\left\|B_{\nu}^{s}\left(f_{\mathbb{P}}^{*}-f_{\mathbf{z}}^{\lambda}\right)\right\|_{\mathcal{H}} \leq C_{r, s, \kappa} \log \left(8 \eta^{-1}\right) \lambda^{s}\left(R\left(\lambda^{r}+\frac{1}{\sqrt{n}}\right)+\left(\frac{M}{n \lambda}+\sqrt{\frac{\sigma^{2} \mathcal{N}(\lambda)}{n \lambda}}\right)\right) .
$$


Proof of Theorem 3.2: Let all assumptions of Theorem 3.2 be satisfied. For proving (3.4) we want to apply Proposition 5.3. Provided $\mathrm{n}$ is big enough, we have $\mathcal{F}\left(\lambda_{n}\right) \geq 1$ and by Lemma 5.2 it holds $\mathcal{N}\left(\lambda_{n}\right) \leq C \cdot \lambda_{n}^{2 r+1} / \mathcal{G}\left(\lambda_{n}\right)$, following from the definition of $\mathcal{G}$. By the definition of $\lambda_{n}$ and by Lemma 5.1. (iii), for n sufficiently large, $\mathcal{G}\left(\lambda_{n}\right) \geq C_{\sigma, R} \frac{1}{n}$, so assumption (5.2) is satisfied if $\log (8 / \eta) \leq C_{\sigma, R} \lambda_{n}^{-r}$. Hence, with probability at least $1-\eta$

$$
\left\|B^{s}\left(f_{\mathbb{P}}^{*}-f_{\mathbf{z}}^{\lambda_{n}}\right)\right\|_{\mathcal{H}} \leq C \cdot \log \left(8 \eta^{-1}\right) \lambda_{n}^{s}\left(R\left(\lambda_{n}^{r}+\frac{1}{\sqrt{n}}\right)+\left(\frac{M}{n \lambda_{n}}+\sigma \sqrt{\frac{\lambda_{n}^{2 r}}{n \mathcal{G}\left(\lambda_{n}\right)}}\right)\right) .
$$

Observe that the choice (3.3) implies that $n^{-\frac{1}{2}}=o\left(\lambda_{n}^{r}\right)$, since $\sigma^{2} / R^{2} n=\mathcal{G}\left(\lambda_{n}\right) \leq \lambda_{n}^{2 r+1}$. Therefore, up to requiring $n$ large enough and multiplying the front factor by 2 , we can disregard the term $1 / \sqrt{n}$ in the second factor of the above bound. Similarly, one can readily check that

$$
\frac{M}{n \lambda_{n}}=o\left(\sqrt{\frac{\lambda_{n}^{2 r}}{n \mathcal{G}\left(\lambda_{n}\right)}}\right)
$$

so that we can also disregard the term $\left(n \lambda_{n}\right)^{-1}$ for $n$ large enough and concentrate on the two remaining main terms of the upper bound in (5.4), which are $R \lambda_{n}^{r}$ and $\sigma \lambda_{n}^{r} \mathcal{G}\left(\lambda_{n}\right)^{-\frac{1}{2}} n^{-\frac{1}{2}}$ - The proposed choice of $\lambda_{n}$ balances precisely these two terms and easy computations lead to (3.4).

5.3. Proof of minimax lower rate. Let $r>0, R>0$ and $s \in[0,1 / 2]$ be fixed. Assume (Eigen $\left.^{>}\left(\nu_{*}\right)\right)$. In order to obtain minimax lower bounds, we apply a general reduction scheme as presented in Chapter 2 of [12]. The main idea is to find $N_{\varepsilon}$ functions $f_{1}, \ldots, f_{N_{\varepsilon}}$ satisfying $\mathbf{S C}(r, R)$, depending on $\varepsilon$ sufficiently small, with $N_{\varepsilon} \rightarrow \infty$ as $\varepsilon \rightarrow 0$, such that any two of these functions are $\varepsilon$-separated with respect to $\|\cdot\|_{s^{-}}$norm, but such that the associated distributions $\mathbb{P}_{f_{j}}=: \mathbb{P}_{j} \in \mathcal{M}$ (see definition (5.6) below) have small KullbackLeibler divergence $\mathcal{K}$ to each other and are therefore statistically close. It is then clear that

$$
\inf _{f_{\bullet}} \sup _{\mathbb{P} \in \mathcal{M}} \mathbb{P}\left[\left\|B_{\nu}^{s}\left(f_{\mathbb{P}}^{*}-f_{\mathbf{z}}\right)\right\|_{\mathcal{H}} \geq \varepsilon\right] \geq \inf _{f_{\bullet}} \max _{1 \leq j \leq N_{\varepsilon}} \mathbb{P}_{j}\left[\left\|B_{\nu}^{s}\left(f_{j}-f_{\mathbf{z}}\right)\right\|_{\mathcal{H}}^{p} \geq \varepsilon\right]
$$

where the infimum is taken over all estimators $f_{\bullet}$ of $f_{\mathbb{P}}^{*}$. The above RHS is then lower bounded through Proposition 6.1 given in supplementary material which is a consequence of Fano's lemma.

We will establish the lower bounds in the particular case where the distribution of $Y$ given $X$ is Gaussian with variance $\sigma^{2}$ (which satisfies $\operatorname{Bernstein}(M, \sigma)$ with $M=\sigma$ ). The main effort is to construct a finite subfamily belonging to the model of interest and suitably satisfying the assumptions of Proposition 5.4 in supplementary. More precisely, to each $f$ satisfying $\mathbf{S C}(r, R)$ and $x \in \mathcal{X}$ we associate the following measure:

$$
\mathbb{P}_{f}(d x, d y):=\mathbb{P}_{f}(d y \mid x) \nu(d x), \text { where } \mathbb{P}_{f}(d y \mid x):=\mathcal{N}\left(f(x), \sigma^{2}\right) .
$$


Then the measure $\mathbb{P}_{f}$ belongs to the class $\mathcal{M}_{(M, \sigma, R)}$, defined in Section 2 , and given $f_{1}, f_{2}$ satisfying $\mathbf{S C}(r, R)$, the Kullback divergence between $\mathbb{P}_{1}$ and $\mathbb{P}_{2}$ satisfies

$$
\mathcal{K}\left(\mathbb{P}_{1}, \mathbb{P}_{2}\right)=\frac{1}{2 \sigma^{2}}\left\|\sqrt{B_{\nu}}\left(f_{1}-f_{2}\right)\right\|_{\mathcal{H}}^{2} .
$$

For the sake of completeness, we give a constitutive result whose proof is given in [12].

Proposition 5.4. Consider a model $\mathcal{P}=\left\{P_{\theta}: \theta \in \Theta\right\}$ of probability measures on a measurable space $(\boldsymbol{Z}, \mathcal{A})$, indexed by $\Theta$. Additionally, let $d: \Theta \times \Theta \longrightarrow[0, \infty)$ be a (semi-) distance. Assume that $N \geq 2$ and suppose that $\Theta$ contains $N+1$ elements $\theta_{0}, \ldots, \theta_{N}$ such that:

(i) For some $\varepsilon>0$, and for any $0 \leq i<j \leq N, d\left(\theta_{i}, \theta_{j}\right) \geq 2 \varepsilon \quad$;

(ii) For any $j=1, \ldots, N, P_{j}$ is absolutely continuous with respect to $P_{0}$, and

$$
\frac{1}{N} \sum_{j=1}^{N} \mathcal{K}\left(P_{j}, P_{0}\right) \leq \omega \log (N)
$$

for some $0<\omega<1 / 8$.

Then

$$
\inf _{\hat{\theta}} \max _{1 \leq j \leq N} P_{j}\left(d\left(\hat{\theta}, \theta_{j}\right) \geq \varepsilon\right) \geq \frac{\sqrt{N}}{1+\sqrt{N}}\left(1-2 \omega-\sqrt{\frac{2 \omega}{\log (N)}}\right)>0,
$$

where the infimum is taken over all estimators $\hat{\theta}$ of $\theta$.

We will need the following Proposition:

Proposition 5.5. For any $\varepsilon>0$ sufficiently small (depending on the parameters $\nu_{*}, r, R, s$ ), there exist $N_{\varepsilon} \in \mathbb{N}$ and functions $f_{1}, \ldots, f_{N_{\varepsilon}} \in \Omega(\nu, r, R)$ satisfying

(1) For any $i, j=1, \ldots, N_{\varepsilon}$ with $i \neq j$ it holds $\left\|B^{s}\left(f_{i}-f_{j}\right)\right\|_{\mathcal{H}}^{2}>\varepsilon^{2}$ and

$$
\mathcal{K}\left(\mathbb{P}_{i}, \mathbb{P}_{j}\right) \leq C_{\nu_{*}, s} R^{2} \sigma^{-2}\left(\frac{\varepsilon}{R}\right)^{\frac{2 r+1}{r+s}}
$$

(2) $\log \left(N_{\varepsilon}-1\right) \geq \frac{1}{36} \mathcal{F}\left(2^{\nu_{*}}\left(\frac{\varepsilon}{R}\right)^{\frac{1}{r+s}}\right)$.

Proof of Proposition 5.5. Assume $\left(\right.$ Eigen $\left.^{>}\left(\nu_{*}\right)\right)$ for any $j \geq j_{0}$, for some $j_{0} \in \mathbb{N}$. Let $\max :=\max \left(28, j_{0}\right)$. Choose $\varepsilon<2^{-\nu_{*}(r+s)} R \mu_{\max }$ and pick $m=m(\varepsilon):=\mathcal{F}\left(2^{\nu_{*}}\left(\varepsilon R^{-1}\right)^{\frac{1}{r+s}}\right)$. Note that $m \geq 28$, following from the choice of $\varepsilon$, so Lemma 6.3 in [1] applies.

Let $N_{m}>3$ and $\pi_{1}, \ldots, \pi_{N_{m}} \in\{-1,+1\}^{m}$ be given by Lemma 6.3 in [1] and define

$$
g_{i}:=\frac{\varepsilon}{\sqrt{m}} \sum_{l=m+1}^{2 m} \pi_{i}^{(l-m)}\left(\frac{1}{\mu_{l}}\right)^{r+s} e_{l} .
$$

We have by the definition of $m$

$$
\left\|g_{i}\right\|_{\mathcal{H}}^{2}=\frac{\varepsilon^{2}}{m} \sum_{l=m+1}^{2 m}\left(\frac{1}{\mu_{l}}\right)^{2(r+s)} \leq \varepsilon^{2} \mu_{2 m}^{-2(r+s)} \leq \varepsilon^{2} 2^{2 \nu_{*}(r+s)} \mu_{m}^{-2(r+s)} \leq R^{2} .
$$


For $i=1, \ldots, N_{m}$ let $f_{i}:=B^{r} g_{i} \in \Omega(\nu, r, R)$, with $g_{i}$ as in (5.10). Then

$$
\left\|B^{s}\left(f_{i}-f_{j}\right)\right\|_{\mathcal{H}}^{2} \geq \varepsilon^{2}
$$

as a consequence of Lemma 6.3 in [1]. For $i=1, \ldots, N_{\varepsilon}$, let $\mathbb{P}_{i}=\mathbb{P}_{f_{i}}$ be defined by (5.6). Then, using the definition of $m$ and (5.7), the Kullback divergence satisfies

$$
\begin{aligned}
\mathcal{K}\left(\mathbb{P}_{i}, \mathbb{P}_{j}\right) & =\frac{1}{2 \sigma^{2}}\left\|\sqrt{B}\left(f_{i}-f_{j}\right)\right\|_{\mathcal{H}}^{2} \leq(2 \sigma)^{-2} \mu_{m+1}^{1-2 s} \varepsilon^{2} \\
& \leq 2^{\nu_{*}(1-2 s)}\left(2 \sigma^{2}\right)^{-1} R^{2}\left(\frac{\varepsilon}{R}\right)^{\frac{1+2 r}{r+s}}
\end{aligned}
$$

which completes the proof of the first part. Finally, again by Lemma 6.3 in [1] and the definition of $m$

$$
\log \left(N_{m}-1\right) \geq \frac{m}{36}=\frac{1}{36} \mathcal{F}\left(2^{\nu_{*}}\left(\varepsilon R^{-1}\right)^{\frac{1}{r+s}}\right)
$$

and the proof is complete.

Proof of Theorem 3.1: Our aim is to apply Proposition 5.4 in supplementary and we will check that all required conditions are satisfied. From Proposition 5.5 we deduce that for any $\varepsilon$ sufficiently small, there exists $N_{\varepsilon}$ and functions $f_{1}, \ldots, f_{N_{\varepsilon}}$ satisfying $\mathbf{S C}(r, R)$ fulfilling points 1 and 2 . The first part of point 1 gives requirement $(i)$ of Proposition 5.4 in supplementary. Requirement ( $i i$ ) follows directly from (5.9) and from point 2 in Proposition 5.5:

$$
\begin{aligned}
\frac{1}{N_{\varepsilon}-1} \sum_{j=1}^{N_{\varepsilon}-1} \mathcal{K}\left(\mathbb{P}_{j}^{\otimes n}, \mathbb{P}_{N_{\varepsilon}}^{\otimes n}\right) & \leq n 36 C_{\nu_{*}, s}^{\prime} R^{2} \sigma^{-2} \mathcal{G}\left(2^{\nu_{*}}\left(\varepsilon R^{-1}\right)^{\frac{1}{r+s}}\right) \log \left(N_{\varepsilon}-1\right) \\
& =: \omega \log \left(N_{\varepsilon}-1\right)
\end{aligned}
$$

with $C_{\nu_{*}, s}^{\prime}=2^{-2 \nu_{*}(r+s)-1}<1$. Define $\varepsilon:=2^{-\nu_{*}} \frac{R}{288} \mathcal{G}^{-1}\left(\frac{\sigma^{2}}{R^{2} n}\right)^{r+s}$, then by Lemma 5.1, the requirements of Proposition Proposition 5.4 in supplementary will hold (in particular, $\omega<1 / 8$ ) for any $n$ sufficiently large and

$$
\inf _{f_{\bullet}} \max _{1 \leq j \leq N_{\varepsilon}} \mathbb{P}_{j}^{\otimes n}\left(\left\|B_{\nu}^{s}\left(f_{\bullet}-f_{j}\right)\right\|_{\mathcal{H}} \geq \frac{\varepsilon}{2}\right) \geq \frac{\sqrt{N_{\varepsilon}-1}}{1+\sqrt{N_{\varepsilon}-1}}\left(1-2 \omega-\sqrt{\frac{2 \omega}{\log \left(N_{\varepsilon}-1\right)}}\right)>0 .
$$

Taking the liminf finishes the proof.

\section{REFERENCES}

[1] G. Blanchard and N. Mücke. Optimal rates for regularization of statistical inverse learning problems. arXiv Preprint (1604.04054), 2016.

[2] A. Caponnetto. Optimal rates for regularization operators in learning theory. Technical report, MIT, 2006.

[3] A. Caponnetto and Y. Yao. Cross-validion based adaptation for regularization operators in learning theory. Analysis and Applications, 8(2):161-183, 2010.

[4] N. Cristianini and J. Shawe-Taylor. Kernel Methods for Pattern Analysis. Cambridge University Press, 2004. 
[5] F. Cucker and S. Smale. Best choices for regularization parameters in learning theory: on the biasvariance problem. Foundations of Computational Mathematics, 2(4):413-428, 2002.

[6] E. De Vito and A. Caponnetto. Optimal rates for regularized least-squares algorithm. Foundations of Computational Mathematics, 7(3):331-368, 2006.

[7] E. De Vito, L. Rosasco, A. Caponnetto, and U. De Giovannini. Learning from examples as an inverse problem. J. of Machine Learning Research, 6:883-904, 2005.

[8] L. Dicker, D. Foster, and D. Hsu. Kernel methods and regularization techniques for nonparametric regression: Minimax optimality and adaptation. Technical report, Rutgers University, 2015.

[9] H. Engl, M. Hanke, and A. Neubauer. Regularization of Inverse Problems. Kluwer Academic Publishers, 2000 .

[10] L. L. Gerfo, L. Rosasco, F. Odone, E. De Vito, and A. Verri. Spectral algorithms for supervised learning. Neural Computation, 20(7):1873-1897, 2008.

[11] C. Stone. Optimal global rates of convergence for nonparametric regression. The Annals of Statistics, 10(4):1040-1053, 1982.

[12] A. Tsybakov. Introduction to Nonparametric Estimation. Springer, 2008.

[13] T. Zhang. Learning bounds for kernel regression using effective data dimensionality. Neural Comput., 17(9):2077-2098, 2005.

Institute of Mathematics, University of Potsdam, Karl-Liebknecht-Strae 24-25 14476 Potsdam, Germany

E-mail address: $\{$ blanchard, nmuecke\}@uni-potsdam.de 